\title{
Anesthetic Considerations in A Case of Arnold Chairi Malformation Type II
}

\author{
Bhavna Gupta* and Anshul Goyal \\ Department of anaesthesia and critical care, Maulana Azad Medical College, New Delhi, India
}

*Corresponding author: Bhavna Gupta, Department of anaesthesia and critical care, Maulana Azad Medical College, Lok nayak hospital, New Delhi, India

\begin{abstract}
Arnold chiari malformation (ACM) Type II is a developmental abnormality of the brainstem and cerebellar tonsil, medulla and protrusion of $4^{\text {th }}$ ventricle into cervical spinal canal. To best of our knowledge, there is paucity of literature regarding anesthetic management of cases with ACM type II. The anesthetic concerns with general anesthesia are related to the risk of autonomic dysfunction, difficult airway management, damage to the spinal cord and sensitivity to neuromuscular blocking agents. There is risk of increasing intracranial pressure and brainstem compression or herniation leading to hemodynamic and respiratory compromise, due to presence of meningomyelocoele, syringohydromyelia and tethered spinal cord.
\end{abstract}

\section{Case Report}

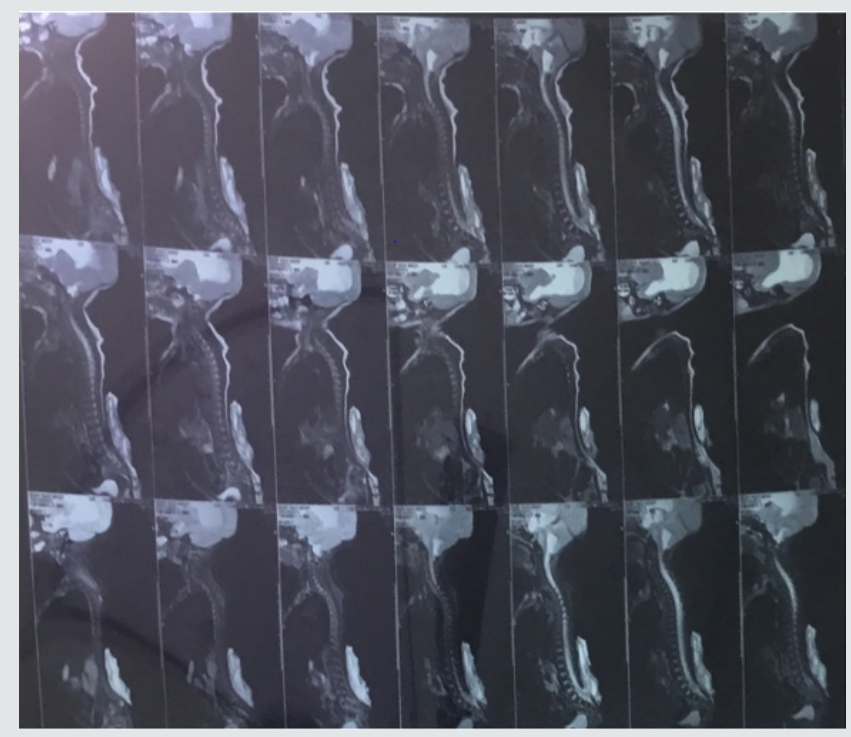

Figure 1: Suggests CT image of spine.

A preterm case of 2 month old female, with diagnosed Arnold chiari malformation type II had gross hydrocephalus. She developed lower respiratory tract infection after one month of birth, and had breath holding spells while feeding. She had lethargy, decreased oral acceptance and was intubated in pediatric emergency in view of respiratory compromise. Ultrasound cranium suggested grossly dilated lateral and third ventricles with evidence of fine internal echoes predominantly in left lateral ventricle. Posterior fossa was shallow and cerebral parenchyma was grossly normal. Magnetic resonance imaging of brain suggested presence of communicating hydrocephalus, syringohydromyelia, pointed peg like cerebellar tonsils with ectopia through the forman magnum upto $\mathrm{C} 1$ vertebra, inferior displacement of brainstem, fourth ventricle and torcular herophilli. There was a low lying posteriorly tethered spinal cord with meningomyeolocele in lumbar region (L4, L5 \&S1). (Figure 1) Child was referred to neurosurgery department in view of features suggestive of raised intracranial pressure, bulging anterior fontanelle, irritability, and intermittent posturing. External ventricular drainage (EVD) was placed under local anesthesia to aspirate cerebrospinal fluid (CSF) and lower intracranial pressure. Drainage of $25 \mathrm{ml}$ of CSF was done every 12 hours but eventually child had episodes of labored rapid shallow breathing and feeble pulses and was intubated in view of the same. She was also started on inotropes (dopamine and adrenaline). Patient was re-intubated again during the hospital stay due to suspected aspiration with respiratory distress, but was extubated after 2 days, after the respiratory efforts became normal. On examination, knee and ankle reflex were absent, plantar were up going, tone was decreased 
in bilateral lower limbs. As EVD was getting blocked and child developed ventriculitis with deteriorating general condition, she was posted to us for placement of ventriculo-peritoneal shunt. After attaching standard monitors, child was induced with inj fentanyl, thiopentone and after checking mask ventilation, inj atracurium was given. Intubation was done by videolaryngoscope in neutral position by an experienced anesthesiologist to prevent neurological damage and to avoid hemodynamic instability associated with neck movement. Maintenance of anesthesia was done with oxygen, nitrous and sevoflurane. Child was extubated uneventfully at the end of surgery and was observed in post-operative anesthesia unit for 24 hours.

\section{Discussion}

Arnold chiari malformation includes group of disorders that are characterized by anatomical anomalies of cerebellum, brainstem, craniocervical junction with downward displacement of cerebellum either alone or together with lower medulla, into spinal canal. ACM is of 4 types-I- IV. ACM type I is the most common type found in children, in which lower part of cerebellum and not the brain stem extends through the base of skull. Type II is seen in children who are born with spina bifida and is also known as the classical type in which both cerebellum and brainstem extend into foramen magnum. Type III is the most serious type and involves the herniation of cerebellum and brainstem through foramen magnum, associated with serious neurological defects. Type IV is incomplete or underdeveloped cerebellum, associated with exposed parts of skull and spinal cord [1]. Other conditions associated with ACM include hydrocephalus, syringomyelia i.e. a cyst in the spinal cord central canal, tethered cord syndrome. ACM II is also associated with Goldenhar syndrome and Albright Hereditary osteodystrophy, connective tissue disorders, pseduohypoparathyroidsm etc. Symptoms of ACM II are because of gross hydrocephalus characterized by repeated episodes of vomiting, irritability seizure, delayed milestones, cranial nerve $9^{\text {th }}$ and $10^{\text {th }}$ dysfunction leading to dysphagia, weakness of vocal cords, swallowing difficulties, breathing irregularities, serious changes in the function of nerves of the throat and tongue (decreased gag reflex) and involuntary ,rapid downward eye movement and respiratory distress, brainstem compression leading to respiratory and hemodynamic compromise. Meningomyelcele is frequently associated with ACM II leading to lack of bladder and bowel control in such children. No two cases of ACM 2 are exactly alike and associated symptoms vary from asymptomatic to life threatening symptoms. Typically patient becomes symptomatic from 2 month to 2 years. Patients presents with symptomatic exacerbations and remissions. Specific symptoms can occur in different combinations generally reflecting dysfunction of cerebellum, brainstem, spinal cord \& lower cranial nerves. Anesthetic considerations with respect to ACM type II include increased intracranial pressure, hydrocephalus, difficult airway, C1-2 instability, requiring neutral intubation, increased risk of hypovolemia because of fluid and blood loss from defects, infection, heat loss from meningomyelocele. There is increased risk of brainstem dysfunction leading to stridor, aspiration, apnea. There is increased risk of autonomic dysfunction leading to arrhythmia, bradycardia, labile blood pressure, sudden respiratory or cardiac arrest. Ventilation-perfusion abnormalities may also be seen in case respiratory muscles are involved. There may be damage to spinal cord because of raised intracranial pressure. These children may also have associated allergy to latex. Impaired thermal regulation is also a concern in such patients. Parents should always be advised that in this or any other neurosurgical case, there is always a chance that the child will remain intubated postoperatively, although the plan is to extubate in the operating room. One should be prepared about cranial nerve and brain stem dysfunction characterized by vocal cord paralysis, apnea, dysphagia and laryngeal stridor in the post-operative period. Goals of anesthesia pertain to avoiding increase in intracranial pressure and maintain cerebral perfusion pressure, latex-free environment and rapid emergence for postoperative neurological examination. Children with ACM type II have remissions of hemodynamic and respiratory compromise, central hypoventilation, shock, requiring meticulous planning and management.

\section{References}

1. Iskandar B, Oakes W (1999) The Chiari Malformations. in Albright A, Pollack I, Adelson P (eds): Principles and Practice of Pediatric Neurosurgery. (1 ${ }^{\text {st }}$ edn). NY: Thieme Medical Publishers. New York, USA.

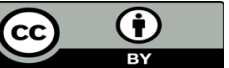

This work is licensed under Creative Commons Attribution 4.0 License

To Submit Your Article Click Here: Submit Article

DOI: $10.32474 /$ GJAPM.2019.01.000106

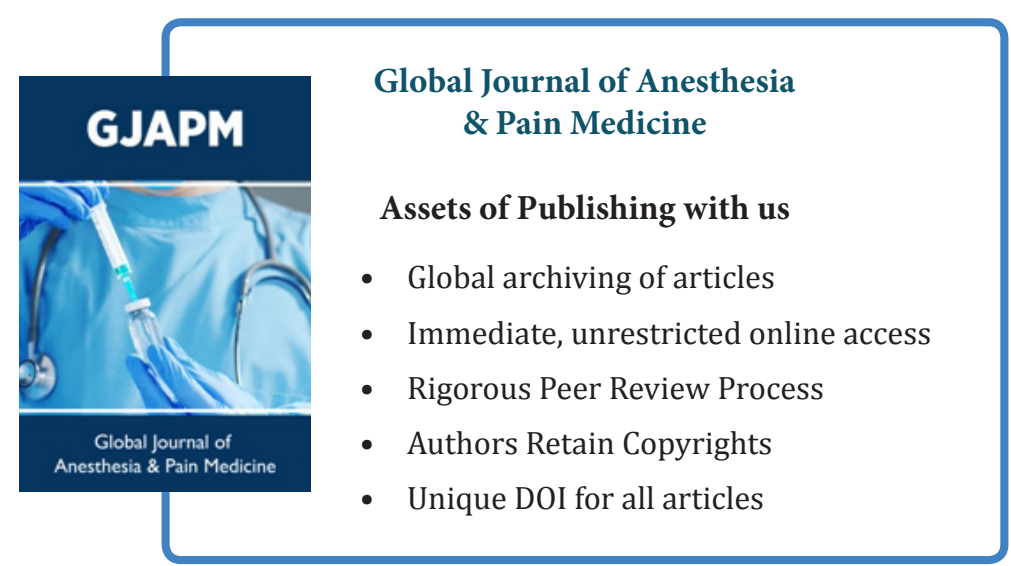

